\title{
Two-tier Graph Contextual Embedding for Cross-device User Matching
}

\author{
Hongren Huang ${ }^{1,2}$, Shu Guo ${ }^{3}$, Chen Li $^{1,2}$, Jiawei Sheng ${ }^{4,5}$, Lihong Wang ${ }^{3}$ * \\ Jianxin $\mathrm{Li}^{1,2}$, Jing $\mathrm{Liu}^{3}$ and Shenghai Zhong ${ }^{1,2}$ \\ ${ }^{1}$ Beijing Advanced Innovation Center for Big Data and Brain Computing, Beihang University, Beijing, China \\ ${ }^{2}$ School of Computer Science and Engineering, Beihang University, Beijing, China \\ ${ }^{3}$ National Computer Network Emergency Response Technical Team/Coordination Center of China \\ ${ }^{4}$ Institute of Information Engineering, Chinese Academy of Sciences, Beijing, China \\ ${ }^{5}$ School of Cyber Security, University of Chinese Academy of Sciences, Beijing, China \\ huanghr@act.buaa.edu.cn,guoshu@cert.org.cn,wlh@isc.org.cn
}

\begin{abstract}
The cross-device user matching task is to identify the behavior-logs (i.e., behavior sequences) on multiple devices that belong to one real person. Due to its anonymous and long-term properties, most previous methods of learning behavior embeddings cannot effectively capture two important features in the sequences, namely high-order connections and long-range dependencies. To this end, we propose a novel framework called Two-tier Graph Contextual Embedding (TGCE) to solve the above problems simultaneously. In the first tier, we construct behavior evolutionary graphs (BEGs) for behavior sequences and design an order-preserving neighbor aggregation network to collectively model transitions of behaviors with their neighbors. As repeated behaviors can be grouped into single nodes, our model joints neighboring environments around behaviors in a collective way, and behavior embeddings can be enriched. In the second tier, we further build scaled shortcut graphs (SSGs) by refining BEGs with random walk-based edge addition, then a position-aware graph attention network is further imposed on SSGs to facilitate fast information propagation. As distant graph nodes can be directly connected by shortcut edges, we can further capture long-range dependencies. By stacking two graph tiers, our approach can obtain graph contextual embeddings for behaviors to further improve user matching. Experimental results on the benchmark dataset show that our model outperforms various baselines in the user matching task. Our code is released on https://github.com/13061051/TGCE_2021.
\end{abstract}

\section{CCS CONCEPTS}

\section{- Information systems $\rightarrow$ Web log analysis.}

\section{KEYWORDS}

User matching; Behavior embedding; Graph neural network

\footnotetext{
${ }^{*}$ Corresponding author. Email:wlh@isc.org.cn
}

Permission to make digital or hard copies of all or part of this work for personal or classroom use is granted without fee provided that copies are not made or distributed for profit or commercial advantage and that copies bear this notice and the full citation on the first page. Copyrights for components of this work owned by others than ACM must be honored. Abstracting with credit is permitted. To copy otherwise, or republish, to post on servers or to redistribute to lists, requires prior specific permission and/or a fee. Request permissions from permissions@acm.org.

CIKM '21, November 1-5, 2021, Virtual Event, QLD, Australia

(c) 2021 Association for Computing Machinery.

ACM ISBN 978-1-4503-8446-9/21/11 ..\$15.00

https://doi.org/10.1145/3459637.3482308

\section{ACM Reference Format:}

Hongren Huang ${ }^{1,2}$, Shu Guo ${ }^{3}$, Chen $\mathrm{Li}^{1,2}$, Jiawei Sheng ${ }^{4,5}$, Lihong Wang ${ }^{3}$ and Jianxin $\mathrm{Li}^{1,2}$, Jing $\mathrm{Liu}^{3}$ and Shenghai Zhong ${ }^{1,2}$. 2021. Two-tier Graph Contextual Embedding for Cross-device User Matching. In Proceedings of the 30th ACM International Conference on Information and Knowledge Management (CIKM '21), November 1-5, 2021, Virtual Event, QLD, Australia. ACM, New York, NY, USA, 10 pages. https://doi.org/10.1145/3459637.3482308

\section{INTRODUCTION}

It's a ubiquitous phenomenon that users' online activities can happen on multiple devices. However, many brands and businesses have inadequate user identities to work with, because users across multiple devices are usually considered different people due to their distinct activities. Automatically detecting the same person across multiple devices is crucial for understanding human behavior patterns, which can support applications like user profiling and online advertising. Owing to the highly practical value, a task named cross-device user matching has quickly gained broad attention in recent years [20]. This task aims to identify all pairs of different device logs that belong to the same users. Here, each device log is formed as a behavior sequence of time-stamped actions taken by a user over a long period of time, say months or even a year. Each action is a behavior item such as a browsing URL. Besides, there is generally no side information (such as user attributes) in the logs due to privacy issues. Both the long-term and anonymous properties pose great challenges to most learning algorithms.

In the past few years, this task has motivated many effective methods developed [20, 24, 34]. The key idea of most approaches is to learn distributed behavior representations (i.e. embeddings) for users using only their historical actions, so as to describe the longterm behavior patterns of users. Early works [24] adopted handcrafted features or unsupervised feature learning methods [13] to compute the representations of behavior sequences. They focused on the co-occurrence of behaviors, thereby capturing shallow connections. Latter deep learning based methods have better capability of expressing dense interactions between behaviors. Tanielian et al. [33] proposed a model named SCEmNet that uses a 2D-CNN (Convolutional Neural Networks) framework to encode representations of behaviors and a siamese network to learn relatedness between two users. But their work mostly captures local interactions in the user behavior sequences, limiting its capacity for learning the whole sequence pattern. Recent studies have highlighted sequential 


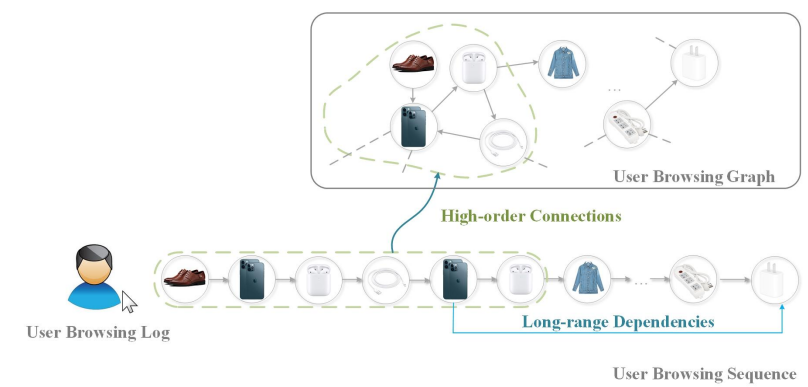

Figure 1: An illustration of a user's behaviors organized by different modes and its two key structural information.

models such as recurrent neural networks (RNNs) and attentionbased methods in modeling sequence patterns, and obtain exciting results in many sequence modeling tasks [8, 23, 26, 30]. Although they work pretty well in the settings of sequence modeling, most of them are not tailored for the user matching task and would be sub-optimal to learn behavior embeddings. There are two major problems worth further investigation.

Firstly, due to the anonymous property, only limited interaction information can be exploited. Most traditional sequential models usually model one-way interactions in a sequence mode, which makes a behavior taking place at different positions interact with its neighbors disjointedly and obtain its respective embedding at each position. This may lead to very sparse behavior interactions. Take the scenario of webpage browsing in Figure 1 as an example. For a user's browsing history, one may browse the item "iPhone" repeatedly. Each "iPhone" associated with diverse contexts will be treated separately in the sequence, resulting in the contexts of each "iPhone" isolated. Actually, the behavior interactions can be denser further, however, in a graph mode. As shown in the figure, the user browsing sequence can be converted to a directed graph, where nodes are unique behaviors, and edges are temporal relations between consecutive behaviors in the sequence. Repeated behaviors can be grouped into single nodes, and then collectively interact with their neighbors within a graph structure. By jointing neighboring environment around behaviors in a collective way, behavior embeddings can be enriched. However, to capture such high-order interactions is still not fully exploited in previous works.

Secondly, behavior sequences in user matching are always long, but capturing long-range dependencies among distant behaviors is almost overlooked. Actually, long-range dependency capturing has been demonstrated to be effective in mining implicit important relationships in various sequence modeling works [2,32], which is also important in our task. Still in the same example in Figure 1, browsing items "iPhone" and "power plug" are distant from each other in the sequence, but they may be still related since users usually browse various related webpages for a long time to get detailed information. The usage of long-range contexts could provide more complementary information and thus assist in enriching contextual information about behaviors. However, a major challenge here is how to devise an effective approach to capture long-range dependencies in a long-term sequence setting.

To tackle the two problems together, we propose a Two-tier Graph Contextualized Embedding method for cross-device user matching, termed as TGCE. The model is formulated as a twotier graph model, and each tier constructs a graph neural network (GNN) to respectively capture high-order connections and longrange dependencies in a joint framework. Specifically, a given user behavior sequence is first converted into a directed behavior evolutionary graph (BEG) whose nodes are the unique behaviors and the edges are the transitions between them. The first tier module draws on the ability of sequential models to obtain graph contextual representations of behaviors, by sequentially aggregating their neighbors in a graph setting. In such a collective manner, both sequence- and graph-patterns are preserved by the behaviors, so as to capture high-order connections in their representations. The second tier module then refines the behavior evolutionary graph via a scaled shortcut mechanism, to further draw global dependencies between distant nodes by adding non-existing edges in an efficient way. As the device logs usually record long-term behaviors, adding all non-existing edges definitely leads to a large computation overhead. For this, we propose a random walk-based shortcut mechanism to alleviate connected graph complexity. Then, a position-aware graph attention network is further designed to capture long-range dependencies between every behavior and its added neighbors. Finally, we apply a readout function to generate a graph-level embedding for each user, and compare the resultant representations to make user matching. This paper makes four key contributions as follows:

- We devise a two-tier GNN model called TGCE for the user matching task, to learn graph contextual behavior embeddings by simultaneously capturing high-order and longrange connections. To our best knowledge, for this task we are the first to enhance representation learning of behaviors in a two-tier graph setting.

- To capture high-order connections, we propose constructing behavior evolutionary graphs (BEGs), and an orderpreserving neighbor aggregation network (ONAN) that captures transitions among neighbouring behaviors.

- To capture long-range dependencies, we design scaled shortcut graphs (SSGs) by refining the BEGs with random walkbased edge addition, and a position-aware graph attention network (PGAT) that effectively propagates information along shortcut connections.

- Extensive experiments on a real-world dataset show that TGCE outperforms various baselines in the user matching task, whether compared to state-of-the-art methods dedicatedly designed to this task or other strong sequential models popularly used in other sequence modeling tasks.

\section{RELATED WORK}

Previous works on the cross-device user matching task usually adopt hand-crafted features. The cross-device user matching task is firstly proposed in the CIKM Cup $2016^{1}$, where the winning teams elaborate hand-crafted features mainly from the visited URLs and times. Lian and Xie [20] selects the top key URLs and divides them into sub-groups to produce fine-grained features. Tay et al. [34] adopts TF-IDF [27] feature of URLs and time features, including hourly patterns and weekly patterns, which takes the first place

\footnotetext{
${ }^{1}$ http://cikm2016.cs.iupui.edu/cikm-cup/
} 
in the competition. Phan et al. [24] further designs SF-ML (Session Frequency Matching Likelihood) feature, which applies a TF-IDFlike weighting scheme for URLs in separated behavior sessions. However, the hand-crafted features do not sufficiently explore more complex semantic information, such as related order of behavior sequence, limiting their performance.

Besides the manually constructed features, the semantic features constructed from the structural information of user behavior data are also essential for the user matching task. Among the methods, Phan et al. [24] applies the Doc2vec method [13] over the user behavior sequences to generate user embeddings. However, they regard Doc2vec embeddings as pre-trained sequential features and do not adapt them to the specific user matching task. To further refine the sequential features, Tay et al. [34] adopts LSTM, and SCEmNet [33] applies 2D-CNN to capture local structures in the behavior sequence for the user matching task.

In addition to the above existing methods, some promising methods construct the supervised behavioral features on recommendation tasks, which may be adjusted to be applied on the user matching task. However, the purpose of behavior modeling in the recommendation system is to predict the items at the next moment by capturing the user's recent behavior patterns. The user matching task is based on the overall behavior pattern of the behavior log data to determine whether the two behavior logs belong to the same natural person. The difference in purpose limits the ability of the models on the user matching task, and some adjustments to the models are required. These models appear in the recommendation task are mainly divided into the sequence based, local graph based, global graph based and matrix based models.

The sequence based models capture the sequential structure features from behavior data to make user behavior embedding. For example, GRU4Rec [8] adopts RNNs to encode the sequential information among behavior items and NARM [16] adopts attention mechanism to capture importance for items. Though the above methods take advantage of sequence-level features, they cannot capture more complex interactions in the user behavior like the high-order connections and the long-range dependencies.

The local graph based methods regard each user's behavior logs as an individual behavior graph, and recommend next-items based on the previous user behaviors. They [4, 25, 38, 39] usually convert each users' behavior logs into a graph, adopt a GNN to update item features, and finally applies a readout function to generate a graph-level embedding. SR-GNN [38] models the behavior sequence as a directed unweighted graph, and then performs graph embedding using a GGNN [18]. LESSR [4] further proposes the edge-order preserving graph for users, which preserves the edgeorder information and shortcut graphs, capturing complex longrange dependencies. Though the local graph based methods achieve promising performance in the recommendation task, they do not explicitly modeling global behavior patterns that are essential for the user matching task. To our best knowledge, our approach TGCE is the first work to explore graph based methods on user behavior modeling, specifically designed for the user matching task.

Global graph based models regard all the behavior items and users as a large entire graph $[10,14,21,31]$ on the recommendation task and other tasks like name recognition, user identity linkage. They construct a heterogeneous graph with all the behavior items and users as nodes, and then learn the user node embeddings as users' behavior representations. For example, MBGCN [10] designs the item-item and user-item propagation layer to capture user embedding in the global graph. There also exist several methods $[7,28]$ in matrix factorization manner, which convert user behavior data into a matrix and generate user embeddings by matrix factorization. Though the global graph based methods and matrix based models can capture global connections between users, they do not explicitly modeling the sequential structure of the user behavior. Besides, the item number in user matching is more than 10 million, making it difficult to construct the global graph or apply matrix factorization on the user-item matrix for the cross-device user matching task.

\section{OUR APPROACH}

In this section, we will give the formulation of the user matching task, and then we describe the proposed model TGCE thoroughly.

\subsection{Problem Definition}

The cross-device user matching task aims to find out which behaviorlogs from different devices would belong to the same person. Formally, for each user $u$, a sequence of actions taken by him/her is denoted as a sequence $S_{u}=\left\langle s_{1}^{u}, s_{2}^{u}, \ldots, s_{n}^{u}\right\rangle$ in time order, where $s_{t}^{u} \in \mathcal{V}_{u}$ represents a behavior item taking place at the $t$-th timestamp, $\mathcal{V}_{u}$ is the set of all unique behavior items and $n$ is the length of the sequence. All users' behavior sequences comprise a set $\mathcal{U}$. Since no additional side information (such as user attributes) is available for privacy concern, our solution is to learn behavior representations only based upon the behavior sequences, in terms of a two-tier graph setting. Figure 2 sketches the overall framework.

Given a user behavior sequence $S_{u}$, the first tier constructs a directed behavior evolutionary graph (BEG). An order-preserving neighbor aggregation network (ONAN) is employed to obtain initial graph contextual behavior embeddings. The second tier is then constructed on top of the first tier. It refines the BEG into a scaled shortcut graph (SSG) by a random walk based shortcut generation mechanism. A position-aware graph attention network (PGAT) is further applied to obtain refined graph contextual behavior embeddings. By stacking two-tier models, our approach can improve behavior representations for users, simultaneously capturing both high-order connections and long-range dependencies. After that, TGCE generates a graph-level representation for each behavior sequence $S_{u}$ by applying a readout function (attention pooling) on its behaviors. A prediction layer is finally used to learn a function $f\left(S_{u}, S_{v}\right)$, which measures the probability of two behavior sequences $S_{u}$ and $S_{v}$ belonging to the same user. According to this function we can figure out whether the two users $(u, v)$ are matched ones. In what follows, we will describe each tier of TGCE in $§ 3.2$ and $\S 3.3$, show how to obtain the sequence embedding in $\$ 3.4$. Last, we give how to perform the prediction and training in $\S 3.5$.

\subsection{The First-tier Graph Neural Network}

The first tier of TGCE is to construct a behavior evolutionary graph for each user's behaviors, and utilizes a graph neural network to model high-order interactions among behaviors in a graph setting.

3.2.1 Construction of Behavior Evolutionary Graph. Recently, modeling sequences in a graph setting has been demonstrated to be 


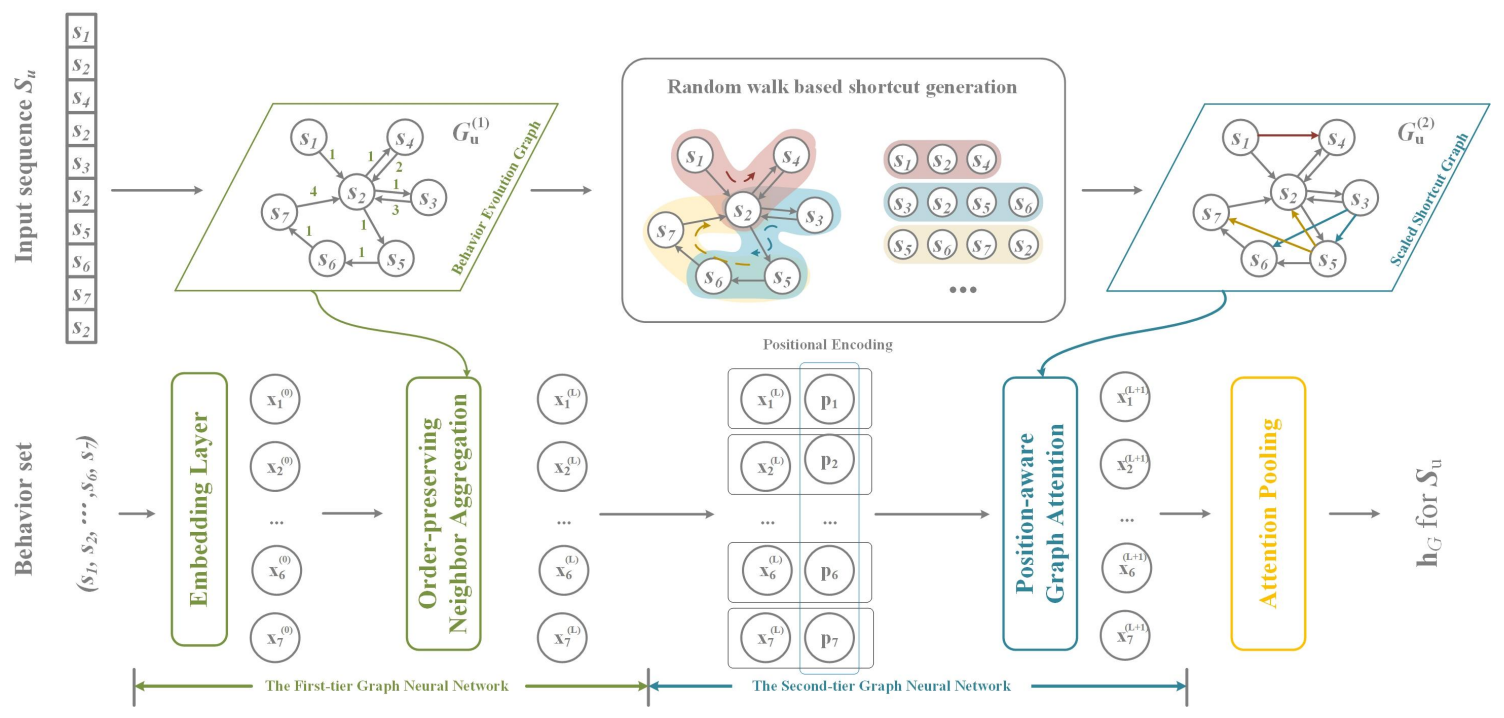

Figure 2: The overview of two-tier GNN model TGCE. Each tier in TGCE is armed with a behavior graph along with a GNN model, so as to produce graph contextual embeddings for behaviors. After stacking two-tier models, TGCE uses attention pooling to obtan a graph-level embedding for each user.

powerful in many sequence modeling tasks, e.g., script event prediction [19] and sequential recommendation [37]. There are various strategies of constructing behavior graphs. Here, we borrow the idea from [4], which provides a lossless sequence-to-graph conversion method by preserving order information in graph edges.

Specifically, given the sequence $S_{u}=\left\langle s_{1}^{u}, s_{2}^{u}, \ldots, s_{n}^{u}\right\rangle$, we take each behavior $s_{t}^{u}$ as a node and each transition $\left(s_{t-1}^{u}, s_{t}^{u}\right)$ as a directed edge which represents a user $u$ takes action $s_{t}^{u}$ after $s_{t-1}^{u}$ in the sequence $S_{u}$. Thus, each sequence for a user $u$ can be defined as a directed graph $G_{u}^{(1)}=\left(\mathcal{V}_{u}, \mathcal{E}_{u}^{(1)}\right)$, where $\mathcal{V}_{u}$ is the set of behavior nodes in $S_{u}$, and $\mathcal{E}_{u}$ is the set of edges. Since several behaviors may appear in the sequence repeatedly in our task, to collectively interact with neighbors, repeated behaviors will grouped into single nodes. To preserve the transition order, all transitions in $S_{u}$ will be retained, and if $\left(s_{t-1}^{u}, s_{t}^{u}\right)$ appears multiple times, we will create multiple edges from $s_{t-1}^{u}$ to $s_{t}^{u}$. Then, for each node $s_{t}^{u}$, its all incoming edges in $\mathcal{E}_{u}^{(1)}$ can be ordered by the time of their occurrences in $S_{u}$. We record the order by assigning a number to each incoming edge in $E_{u}^{(1)}$ to indicate its relative order. For example, the incoming edge appears first in $\mathcal{E}_{u}^{(1)}$ is given 1, the next edge in $\mathcal{E}_{u}^{(1)}$ is given 2. Figure 2 also illustrates the construction of $G_{u}^{(1)}$. We call this resultant graph as the behavior evolutionary graph (BEG). Unlike behavior pairs or behavior sequences, BEG has dense connection patterns among behaviors and contains more abundant interaction information. Next, we will apply a graph neural network on BEG to encode such graph patterns in the representations of behaviors.

3.2.2 Order-preserving Neighbor Aggregation Network. Given a BEG $G_{u}^{(1)}$, we need to properly handle behaviors and their associated neighbors so that the transition order can be well preserved. Most existing GNNs such as GCN (Graph Convolution Network) [12] and GAT (Graph Attention Network) [36] usually use permutation-invariant aggregation functions, which limit them in modeling the order information of edges. Therefore, a GNN which can preserve temporal order information in graphs is required.

Specifically, for a target behavior node $s_{i}$ in $\mathcal{V}_{u}$, let $E_{\text {in }}(i)=$ $\left\langle\left(s_{j_{1}}, s_{i}\right),\left(s_{j_{2}}, s_{i}\right), \cdots,\left(s_{j_{m}}, s_{i}\right)\right\rangle$ denote the ordered list of incoming edges of node $s_{i}$, and $N_{i n}(i)=\left\langle s_{j_{1}}, s_{j_{2}}, \cdots, s_{j_{m}}\right\rangle$ lists the source nodes in $E_{i n}(i) . m$ is the in-degree of the node $s_{i}$. Then, all neighboring nodes in $N_{i n}(i)$ and $s_{i}$ itself (denoted as $\widetilde{N}_{i n}(i)=\left\langle s_{j_{1}}, s_{j_{2}}, \cdots, s_{j_{m}}\right.$, $\left.s_{i}\right\rangle$ ) will be sequentially fed into an order-preserving neighbor aggregation network (ONAN). It will employ a sequential model to aggregate the information passed from neighboring nodes to the target node $s_{i}$, which can be defined recursively as follows:

$$
\begin{gathered}
\mathbf{x}_{i}^{(l+1)}=\operatorname{Upd}^{(l)}\left(\mathbf{x}_{i}^{(l)}, a g g_{i}^{(l)}\right), \\
a g g_{i}^{(l)}=\operatorname{Agg}^{(l)}\left(\left\{\mathbf{x}_{j_{k}},{ }^{(l)}: s_{j_{k}} \in N_{i n}(i)\right\}, \mathbf{x}_{i}^{(l)}\right) .
\end{gathered}
$$

where $\mathbf{x}_{j_{k}}^{(l)} \in \mathbb{R}^{d}$ and $\mathbf{x}_{i}^{(l)} \in \mathbb{R}^{d}$ are the vector embeddings of behavior nodes $\left(s_{j_{k}}\right.$ and $\left.s_{i}\right)$ at $l$-th layer, representing their latent semantics. We denote the total number of layers in the first-tier GNN as $L$. The aggregation function $\operatorname{Agg}(\cdot)$ is used to aggregate the neighborhood information passed to the target node $s_{i}$. The updating function $\operatorname{Upd}(\cdot)$ updates the node representations with the aggregated information. After $L$ steps of information propagation along the edges, the final node representations in the last layer $\mathbf{x}_{i}^{(L)}$ (denoted as $\mathbf{x}_{i}^{\prime}$ ) could capture the information about the local graph structure within a $L$-hop neighborhood community.

Here, GRU (Gated Recurrent Unit) is applied as $\operatorname{Agg}(\cdot)$ to model the relative order information of neighbors $\widetilde{N}_{i n}(i)$ as follows:

$$
\mathbf{h}_{k}^{(l)}=\operatorname{GRU}\left(\mathbf{W}_{G}^{(l)} \mathbf{x}_{j_{k}}, \mathbf{h}_{k-1}^{(l)}\right),
$$

where $\left\{\mathbf{h}_{k}^{(l)}: 0 \leq k \leq m+1\right\}$ are the hidden states of GRU, and the initial state $\mathbf{h}_{0}^{(l)}$ is set to a zero vector; $\mathbf{W}_{G}^{(l)} \in \mathbb{R}^{d \times d}$ is the layer-specific trainable weight matrix. Then, we simply use a 
linear transformation function defined for $\operatorname{Upd}(\cdot)$ to update the representation of the target node $s_{i}$ :

$$
\mathbf{x}_{i}^{(l+1)}=\mathbf{W}_{u}^{(l)} \cdot\left[\mathbf{x}_{i}^{(l)} \mid \mathbf{h}_{i}^{(l)}\right]
$$

where $\mathbf{W}_{u}^{(l)} \in R^{2 d \times d}$ are learnable matrix parameters; [ $\left.\cdot \cdot \cdot\right]$ denotes the concatenation operation.

The above process shows that the first-tier GNN model ONAN could capture rich local contextual information of adjacent behaviors via neighbor aggregation in the entire behavior sequence. Since the edges $E_{i n}(i)$ are ordered chronologically in the BEG, our aggregator will perform the aggregation in a fixed order and then preserve their sequential transition patterns.

\subsection{The Second-tier Graph Neural Network}

Like most GNN models, ONAN can propagate information in each layer to capture 1-hop relations, and further capture global graph structures by stacking multiple layers. However, stacking multiple layers does not necessarily increase performance due to the overfitting and over-smoothing problems [15]. That is why most GNN-based methods, including our ONAN, can capture local context information but cannot effectively solve the long-range dependency problem. To handle this problem, Chen and Wong [4] first proposes LESSR that uses GAT to propagate information on shortcut graphs which connect all node pairs directly. But their work is specifically designed for session-based recommendation, where a session is a sequence of items in close temporal proximity, even no longer than 5 in their work. It's unpractical to construct such shortcut graphs in our long-term behavior modeling setting, where the length of a sequence is commonly very large, say 200. Adding all shortcut edges obviously leads to a large computation overhead. In addition, connecting all node pairs directly will introduce many noise relations and lose the capability of representing the graph structure. All in all, not all node pairs should be connected by shortcut edges. A challenge here is seeking a graph construction approach for a graph model that combines the advantages of scalability and effectiveness, one which can capture dependencies at long range with low computational complexity.

3.3.1 Construction of Scaled Shortcut Graph. To this end, we propose scaled shortcut graphs (SSGs) by refining BEGs with random walk-based shortcut edge addition. The random walk strategy is a technique widely applied in graph embedding [6] to capture graph structural properties, and has been shown to be scalable to large graphs [22]. Here, we will use this strategy to generate shortcuts.

Given a sequence $S_{u}$ with its own BEG $G_{u}^{(1)}$, we create a SSG $G_{u}^{(2)}=\left(\mathcal{V}_{u}, \mathcal{E}_{u}^{(2)}\right)$ whose nodes are the same as $G_{u}^{(1)}$, and edges $\mathcal{E}_{u}^{(2)}$ include original edges $\mathcal{E}_{u}^{(1)}$ and shortcut edges added by the following steps. For each node in $G_{u}^{(1)}$, we perform random walks along the directed edges in $\mathcal{E}_{u}^{(1)}$ to generate node sequences. A random walk of length $H$ starting from a behavior node $s_{i} \in \mathcal{V}_{u}$ consists of a behavior sub-sequence $W\left(s_{i}\right)=\left\{s_{i}, s_{w_{1}}, s_{w_{2}}, \cdots, s_{w_{H}}\right\}$, where $s_{w_{j}} \in \mathcal{V}_{u}$ represents the node index at the $j$-th position in the walk. In general, the $j$-th node in a random walk is generated by sampling a node $s_{w_{j}}$ given previous nodes from a transition probability distribution. Here, for simplicity, we get a next node by uniformly sampling one from the current node's neighbors:

$$
p\left(s_{w_{j}} \mid s_{w_{j-1}}\right)=\frac{1}{\left|\mathcal{N}_{\text {out }}\left(s_{w_{j-1}}\right)\right|},
$$

where $\mathcal{N}_{\text {out }}\left(s_{w_{j-1}}\right)$ denotes 1-hop outgoing neighbors of node $s_{w_{j-1}}$ in $G_{u}^{(1)}$, and $\left|\mathcal{N}_{\text {out }}\left(s_{w_{j-1}}\right)\right|$ is its out-degree. The transition probability is non-zero only if there is an edge from node $s_{w_{j-1}}$ to node $s_{w_{j}}$. Such a random walk will be performed $K$ times for each node, so as to traverse the global graph as far as possible. Then, for any sampled random walk, we create a new edge for each ordered pair of nodes $\left(s_{i}, s_{w_{j}}\right)$, if and only if $s_{w_{j}} \in W\left(s_{i}\right)$. After performing random walks on all nodes, these newly generated edge sets are added to the original BEG $G_{u}^{(1)}$ to form a new denser graph. The resultant graph is the scaled shortcut graph $G_{u}^{(2)}$. Figure 2 shows an illustration of constructing a SSG.

Our shortcut generation method requires $O(N H K)$ in time complexity, scaling linearly to $N, H$, and $K$. Here, $N$ denotes the number of nodes in $\mathcal{V}_{u}$. Usually, $H$ and $K$ are much less than $N$. Compared to the traditional shortcut generation method LESSR that generates shortcuts for all node pairs (which requires $O\left(N^{2}\right)$ ), our approach can be more efficient to handle large-scale behavior graphs. Besides, our shortcuts are generated by random walks rather than choosing all node pairs. Thus, node pairs that appear close to each other in the walk help find those shortcuts compatible with the graph structure. Next, we will apply another graph neural network on SSG to perform fast information propagation along shortcut connections, further capturing dependencies at long range.

3.3.2 Position-aware Graph Attention Network. The shortcut edges help distant nodes to be directly connected in the graph, without going through intermediate nodes. We then design an attention-based architecture based on GAT to model their shortcut connections, so as to capture the long-range dependencies. Compared to sequence models like LSTM [9] and GRU [5], a disadvantage of self-attention mechanisms is that the order information is lost, which however is greatly important to the sequence modeling tasks like user matching. To preserve behavior evolutionary nature in GAT, we propose position-aware GAT (PGAT), which adds positional encoding to GAT in order to inject position information into the attention layer.

For a behavior node $s_{i}$ in $G_{u}^{(2)}$, the PGAT uses the following attention mechanism to propagate information along (original and shortcut) edges in the SSGs and update node representations:

$$
\begin{gathered}
\mathbf{x}_{i}^{\prime \prime}=\sum_{s_{j} \in \mathcal{N}_{i n}(i)} \alpha_{i j} \mathbf{W}_{r} \mathbf{x}_{j}^{\prime} \\
\alpha_{i j}=\frac{\exp \left(e_{i j}\right)}{\sum_{s_{k} \in \mathcal{N}_{i n}(i)} \exp \left(e_{i k}\right)} \\
e_{i j}=\mathbf{q}_{1}^{T} \operatorname{Relu}\left(\mathbf{W}_{a}\left[\mathbf{W}_{q} \mathbf{x}_{i}^{\prime}\left|\mathbf{W}_{k} \mathbf{x}_{j}^{\prime}\right| \mathbf{p}_{i} \mid \mathbf{p}_{j}\right]\right)
\end{gathered}
$$

where $\mathbf{x}^{\prime}$ is the final representation outputted by the first-tier model defined in Eq. (4); $\mathcal{N}_{i n}(i)$ is the set of neighbor nodes pointing to the target node $s_{i} ; \alpha_{i j}$ denotes the attention score of a neighbor $s_{j}$; $\mathbf{q}_{1} \in R^{d}$ is a column vector, $\mathbf{W}_{a} \in R^{d \times\left(2 d+2 d_{p}\right)}$ and $\mathbf{W}_{q}, \mathbf{W}_{k}, \mathbf{W}_{r} \in R^{d \times d}$ are learnable parameters; and ReLU is the activation function [1]. $\mathbf{p}$ is a position encoder to generate an embedding for a node, which encodes the information about the position where the node first appears in the sequence $S_{u}$. There are 
many choices of positional encoders [29, 35, 40]. In this work, we borrow a popular idea from [35], and use sine and cosine functions of different frequencies:

$$
\begin{gathered}
{\left[\mathrm{p}^{\text {pos }}\right]_{2 k}=\sin \left(p o s / 10000^{2 k / d_{p}}\right)} \\
{\left[\mathrm{p}^{\text {pos }}\right]_{2 k-1}=\cos \left(p o s / 10000^{2 k / d_{p}}\right)}
\end{gathered}
$$

where pos is the position, $[\cdot]_{k}$ takes the $k$-th dimension of the vector $\left(k=1, \cdots, d_{p} / 2\right)$, and $d_{p}$ is the dimensional size of the position embedding space. Through this function, our model can easily obtain the positions of nodes without further learning.

The second-tier GNN model PGAT can rapidly capture longrange dependencies because it propagates information along the shortcut connections between nodes merely in one step. Then, it can be combined with the first tier (i.e., ONAN) to increase its capability of capturing global dependencies. Also note that we can stack multiple PGAT layers like ONAN to implicitly capture multi-hop relationships. But we need not to do that, since modeling 1-hop relationships between nodes in SSGs is enough to explicitly capture multi-hop relationships, and particularly will be parameterefficient for training. Actually, ONAN and PGAT can be combined in different orders of layers. We suggest to put the ONAN layer in front of the PGAT layer. PGAT models long-rang dependencies by introducing shortcut connections, but these connections are not necessarily true relationships. It's difficult to distinguish true or false relationships based on initial behavior representations. This will make the neighbor aggregation process in PGAT noise-filled. Thus, it's better to perform behavior embedding first by ONAN, which preserves the original graph structure.

\subsection{Behavior Sequence Embedding}

After stacking two tier GNN models one by one, we can obtain the final representation for each node $s_{i} \in \mathcal{V}_{u}$ as follows:

$$
\mathrm{g}_{i}=\mathbf{W}_{c}\left[\mathbf{x}_{i}^{0} \| \mathbf{x}_{i}^{\prime \prime}\right]
$$

where $\mathbf{x}_{i}^{0}$ is the initial embedding; $\mathbf{x}_{i}^{\prime \prime}$ is the final embeddings obtained from the second-tier GNN model; and $\mathbf{W}_{c} \in R^{d \times 2 d}$ is a learnable matrix parameter.

To obtain a global embedding vector for behavior sequence $S_{u}$, we apply a readout function to construct a graph-level embedding. We adopt an attention-pooling mechanism to the behaviors in $S_{u}$, as we believe that different behaviors may have different weights for representing the sequence. By calculating the relative importance of each behavior node, the graph-level representation $\mathbf{h}_{G}$ for $S_{u}$ can be generated as follows:

$$
\begin{gathered}
\mathbf{h}_{G}=\sum_{s_{i} \in \mathcal{V}_{u}} \beta_{i} \mathbf{g}_{i} \\
\beta_{i}=\frac{\exp \left(\epsilon_{i}\right)}{\sum_{s_{k} \in \mathcal{V}_{u}} \exp \left(\epsilon_{k}\right)} \\
\epsilon_{i}=\mathbf{q}_{2}^{T} \mathbf{g}_{i}+b
\end{gathered}
$$

where $\mathbf{q}_{2} \in R^{d}$ and $b \in R$ are learnable parameters. The graphlevel representation learned by this way could capture the global properties of the entire sequence.

\subsection{User Matching and Training}

After obtaining graph-level representations for users, we can compare two users by computing a similarity of two user's behavior sequences. For any two users $u$ and $v$, we fed the representations into a deep neural network to measure the probability of two behavior sequences $S_{u}$ and $S_{v}$ belonging to the same user:

$$
f\left(S_{u}, S_{v}\right)=\operatorname{DNN}\left(\mathbf{h}_{G_{u}} \otimes \mathbf{h}_{G_{v}}\right)
$$

where $\mathbf{h}_{G_{u}}$ and $\mathbf{h}_{G_{v}}$ are the graph-level representations for $S_{u}$ and $S_{v}$, defined in Eq.(12); and $\otimes$ denotes an element-wise multiplication. DNN (Deep Neural Network) is implemented by a multi-layer fullyconnected neural network followed by a sigmoid function, where each hidden layer performs the following computation:

$$
\mathbf{a}^{(l+1)}=\sigma\left(\mathbf{W}^{(l)} \mathbf{a}^{(l)}+\mathbf{b}^{(l)}\right), l=1, \cdots, M,
$$

where $l$ is the layer number and $M$ is the total number of layers; $\sigma$ is the Relu function; $\mathbf{a}^{(l)}, \mathbf{b}^{(l)}$, and $\mathbf{W}^{(l)}$ are the activation, bias, and model weight at the $l$-th layer. The initial activation $\mathbf{a}^{(0)}=\mathbf{h}_{G_{u}} \otimes \mathbf{h}_{G_{v}}$, and the last prediction value $\mathbf{a}^{(M)}$ will be fed into the sigmoid function $g(x)=1 /(1+\exp (-x))$ to obtain the final similarity. Actually, our approach can also support various similarity metrics as the relatedness score $f\left(S_{u}, S_{v}\right)$, such as cosine similarity, dot similarity and euclidean similarity. We choose a deep neural network because of its capacity of capturing dense connections.

our model then adopts minimizing the Binary Cross Entropy loss function as the training objective:

$$
L=-\sum_{u, v \in \mathcal{U}}\left(y_{u, v} \log f\left(S_{u}, S_{v}\right)+\left(1-y_{u, v}\right) \log \left(1-f\left(S_{u}, S_{v}\right)\right)\right)
$$

where $y_{u, v}$ is the ground-truth of a user pair $(u, v)$, with $y_{u, v}=1$ if $(u, v)$ belongs to the same person and $y_{u, v}=0$ otherwise. Then, all trainable parameters in TGCE are randomly initialized and learned in the back-propagation training paradigm. All behavior node embeddings are initialized by a pre-trained model and will be fixed during training to avoid overfitting.

\section{EXPERIMENTS AND ANALYSIS}

This section presents our experiments and results. We first describe the experimental settings. After that, we conduct extensive analysis with ablation study and parameter study.

\subsection{Dataset}

We conduct experiments on a benchmark dataset publicly released online, ${ }^{2}$ which is a subset of CIKM Cup 2016 competition dataset provided by Data Centric Alliance (DCA). DCA contains anonymized and distinct browsing logs from different devices within 2 months. Each device-log (i.e., behavior sequence) is a list of behaviors in the form of a tuple $\langle U R L$, time $\rangle$, representing the address of a website and the visiting time. URL is in form of a multi-level string "a/b/c/..., where $a, b$, and c are hash-codes of words in the address. There are no additional webpage contents in the URLs. The total number of URLs in DCA is 14,148,535, and the average number of URLs per device-log is 197. Such a dataset reflects the long-term property of the user matching task. The dataset is further divided into training/testing set with 50,146/48,122 device-logs in each one.

\footnotetext{
${ }^{2}$ https://drive.google.com/drive/folders/0B7XZSACQf0KdNXVIUXEyVGlBZnc
} 
There are 107,654 and 107,653 golden links in each set, where a golden link means a pair of device-logs from the same user.

\subsection{Baselines}

We compare the performance of our approach against three groups of methods as baselines. The first group includes representative techniques adopted in existing user matching works.

- TF-IDF is a statistic method which adopts TF-IDF features commonly used by traditional user matching methods [24, 34]. The method counts the frequency of each token in behavior sequences to construct TF-IDF vectors for users.

- Doc2vec learns document embeddings via the distributed memory and distributed bag of words. The method in [24] treats each behavior sequence as a document and uses Doc2vec to learn distributed representations of behaviors.

- SCEmNet [33] applies 2D-CNN to learn the embeddings of behaviors. It is also the state-of-the-art solution applied in the user matching task.

In the second group, we directly compare with two popular sequential models i.e., GRU and Transformer. In the third group, we adapt two strong GNN models in our task, which are originally proposed for session-based recommendation, i.e., SR-GNN and LESSR. Both two methods model behavior sequences in a graph setting.

- SR-GNN [38] transforms behavior sequences into directed unweighted graphs and extract behavior features using a GGNN [18]. But in this work, the relative order among neighboring behaviors is losing in the graphs.

- LESSR [4] converts behavior sequences into two different graphs that can preserve both order information and longrang dependencies. Then it alternates two kinds of GNN layers for multiple steps to obtain behavior embeddings.

For all compared models, we use identical DNN layer (defined in Eq.(15)) to calculate the similarity between user pairs based on their behavior embeddings, so as to make user matching.

\subsection{Evaluation Protocol}

We evaluate all approaches under two different evaluation settings.

(1) User pair classification: This task is originally proposed in the CIKM 2016 Cup. It aims to find really matched user pairs as many as possible from all user pairs in DCA. The task can measure the model's ability to find matched user pairs from a global perspective. To avoid explosive searching, following the protocol in [24], we first limit a candidate set for each device-log to the union of 30 nearest device-logs via cosine similarity based on TF-IDF and Doc2vec features. Then we select from all candidate sets the user pairs $(u, v)$ satisfying their behavior sequence similarity higher than a threshold like $f\left(S_{u}, S_{v}\right)>0.5$ as the final matched users.

(2) User ranking: We also construct another task referring to the setting of user identity linkage across social networks [17]. The User ranking experiment evaluates the matching results for each local user, which can provide more fine-grained indicator to assess the ranking ability of models. For one user $u$ with its positive pairs $(u, v)$, we first corrupt the other user $v$ and replace it by a candidate set. Another 19 nearest device-logs are created as negative examples
Table 1: Results of different models under two settings.

\begin{tabular}{l|ccc|cccc}
\hline & \multicolumn{3}{|c|}{ Classification } & \multicolumn{4}{c}{ Ranking } \\
\cline { 2 - 8 } & Precision & Recall & F1 & HR@3 & HR@5 & HR@10 & MRR@10 \\
\hline TF-IDF & 0.328 & 0.267 & 0.261 & 0.750 & 0.842 & 0.941 & 0.647 \\
Doc2vec & 0.290 & 0.205 & 0.240 & 0.828 & 0.870 & 0.934 & 0.784 \\
SCEmNet & 0.381 & 0.444 & 0.410 & 0.798 & 0.874 & 0.950 & 0.705 \\
\hline GRU & 0.368 & 0.489 & 0.420 & 0.826 & 0.896 & 0.961 & 0.737 \\
Transformer & 0.389 & 0.474 & 0.428 & 0.830 & 0.897 & 0.960 & 0.744 \\
\hline SR-GNN & 0.347 & 0.337 & 0.342 & 0.752 & 0.839 & 0.932 & 0.656 \\
LESSR & 0.407 & 0.480 & 0.440 & 0.850 & 0.911 & 0.967 & 0.765 \\
\hline TGCE & $\mathbf{0 . 4 1 7}$ & $\mathbf{0 . 5 1 3}$ & $\mathbf{0 . 4 6 0}$ & $\mathbf{0 . 8 6 4}$ & $\mathbf{0 . 9 1 8}$ & $\mathbf{0 . 9 6 9}$ & $\mathbf{0 . 7 8 5}$ \\
\hline
\end{tabular}

based on the above-mentioned similarity measure. The task aims to recommend a matched user with the highest similarity.

To evaluate the model's performance, we choose the standard metrics of Precision, Recall and F1 in the user pair classification experiment. The metrics reflect a model's ability of distinguishing matched user pairs from the remaining testing set. For the second task, following the user identity linkage task, we adopt the commonly used HR@N (Hit Rate, $N=3,5,10$ ) and MRR@10 (Mean Reciprocal Rank) as evaluation metrics. They evaluate a model's ability of completing user pairs with one user missing.

\subsection{Implementation Details}

We preprocess the dataset following previous works [24]. To capture URL matching at different granularities, each URL is converted into four terms according to the level of URL. For example, the URL "a/b/c/d/..." can be represented as "a", "a/b", and "a/b/c", "a/b/c/d/..." in different four levels. By applying this strategy, each original device-log will be converted into four sequences. Each sequence will be fed into user matching models to generate a sequence embedding. We then concatenate four embeddings into one vector to generate the final representation for each user.

We implement all baselines based on the codes released by previous literature. For a fair comparison in all compared methods, we tune the embedding size $d$ in the range of $\{16,32,64,128\}$. We adopt Adam Optimizer [11] to optimize all embedding-based models and the initial learning rate is tuned in range of $\left\{10^{-1}, 10^{-2}, 10^{-3}, 10^{-4}\right\}$. The L2 regularization is further used to avoid overfitting and its coefficient is tuned in $\left\{10^{-3}, 10^{-4}, 10^{-5}, 10^{-6}\right\}$. For each positive user pair, we construct negative pairs (non-overlapping with positive ones) during each training epoch, with a positive-to-negative ratio of 1:3. To make the negatives as difficult as possible, we follow the sampling method introduced by [24], and find similar user pairs with the cosine similarity based on TF-IDF and Doc2vec features. We further set the maximum length of each walk to $H=10$, and the number of random walks per behavior node is set to $K=2$, by considering a trade-off between the performance and efficiency. For the baseline LESSR, we also restrict the maximum length of shortcut edges to 10 to avoid computational explosion problems.

We adopt grid search to find the optimal hyperparameters by using $5 \%$ of the training set as the validation set. All experiments for comparing methods are repeated 5 times by drawing new training/validation splits, and compute average as results. Best hyperparameters are selected by early stopping on the validation (monitoring F1 or MRR@10), with at most 100 training iterations. 
Table 2: Results of combining hand-crafted features.

\begin{tabular}{l|ccc}
\hline \multirow{2}{*}{} & \multicolumn{3}{|c}{ Classification } \\
\cline { 2 - 4 } & Precision & Recall & F1 \\
\hline GBUL & 0.786 & 0.551 & 0.648 \\
SCEmNet & 0.795 & 0.558 & 0.656 \\
\hline GRU & 0.790 & 0.556 & 0.653 \\
Transformer & 0.793 & 0.556 & 0.654 \\
\hline SR-GNN & 0.734 & 0.534 & 0.619 \\
LESSR & 0.787 & $\mathbf{0 . 5 6 3}$ & 0.657 \\
\hline TGCE & $\mathbf{0 . 8 0 4}$ & $\mathbf{0 . 5 6 3}$ & $\mathbf{0 . 6 6 3}$ \\
\hline
\end{tabular}

\subsection{Performance Comparisons}

4.5.1 Comparing Behavior Sequence Features. Table 1 shows the results in two experiment setting respectively. From the results, we have the following observations:

(1) Our model TGCE consistently outperforms all baselines in all the metrics, including the existing user matching methods, strong sequential models and graph based models. This reveals the superiority of our approach.

(2) TGCE performs better than the two conventional feature learning methods (TF-IDF and Doc2Vec) by a large margin. These two baselines learn feature vectors for sequences simply based on the behavior co-occurrence information, without considering important sequential information. This definitely produces poor results compared to those deep learning techniques that are capable of modeling long-term sequences.

(3) TGCE can also beat very strong sequence embedding models like SCEmNet, GRU and Transformer, where the improvements mainly come from the capability of simultaneously capturing highorder connections and long-range dependencies. The traditional sequential models such as SCEmNet and GRU can only model behaviors in an one-way manner, where behaviors with their neighbors in different positions will be modeled disjointedly. Transformer is able to draw global connections by its self-attention network. However, it disperses the distribution of attention, resulting in lacking local dependencies over adjacent items and limiting its capacity for learning contextualized representations. In contrary, our TGCE learns graph contextual representations for behaviors by modeling behaviors with their neighbors (connected either by original or shortcut edges) in a collective manner, so as to capture denser connections among behaviors. The results further indicate the effectiveness of modeling sequences in a graph setting.

(4) SR-GNN and LESSR, although powerful in session-based recommendation, show inferior performance than TGCE in user matching. SR-GNN cannot well preserve order information in the graph, which degenerates the matching performance substantially. LESSR solves this problem and obtains very promising results, but it would like to generate sequence representations which focuses more on local behavior patterns. It demonstrates our model's superiority of modeling sequential and global behavior patterns.

4.5.2 Combining Hand-crafted Features. Some previous user matching works may combine learned behavior sequence embeddings with some elaborately hand-crafted features [24,34], such as hourly time patterns, to improve user matching performance. We also conducted such experiments in Table 2. GBUL [24] combines two kinds of sequence-level features as in TF-IDF and Doc2vec with the hand-crafted features into an XGBoost classifier [3] to compute
Table 3: Results of TGCE variants.

\begin{tabular}{l|ccc|cccc}
\hline & \multicolumn{3}{|c|}{ Classification } & \multicolumn{4}{c}{ Ranking } \\
\cline { 2 - 8 } & Precision & Recall & F1 & HR@3 & HR@5 & HR@10 & MRR@10 \\
\hline w/o PGAT & $\mathbf{0 . 4 3 7}$ & 0.475 & 0.456 & 0.856 & 0.914 & 0.965 & 0.778 \\
w/o ONAN & 0.396 & 0.470 & 0.429 & 0.859 & 0.916 & $\mathbf{0 . 9 7 1}$ & 0.776 \\
\hline repl. GCN & 0.401 & 0.491 & 0.442 & 0.850 & 0.909 & 0.965 & 0.770 \\
repl. GAT & 0.384 & 0.529 & 0.445 & 0.859 & 0.915 & 0.967 & 0.781 \\
\hline repl. Cos & 0.070 & $\mathbf{0 . 6 9 4}$ & 0.127 & 0.515 & 0.630 & 0.803 & 0.448 \\
repl. Dot & 0.116 & 0.232 & 0.194 & 0.324 & 0.457 & 0.694 & 0.281 \\
\hline TGCE & 0.417 & 0.513 & $\mathbf{0 . 4 6 0}$ & $\mathbf{0 . 8 6 4}$ & $\mathbf{0 . 9 1 8}$ & 0.969 & $\mathbf{0 . 7 8 5}$ \\
\hline
\end{tabular}

the similarity of user pairs. We directly report GBUL's experiment results from its paper [24]. Except for Doc2vec and TF-IDF, the other behavior representation models, including our model, are supervised and neural network-based models. XGBoost is not a neural network-based model and cannot apply the supervised information to train the supervised behavior embedding methods. Therefore, we use a neural network layer instead of XGBoost as the classifier for these methods. We combined the hand-crafted features on the DNN layer defined in Eq.(15), and Eq.(15) is then changed as $f\left(S_{u}, S_{v}\right)=\operatorname{DNN}\left(\left[\left(h_{G_{u}} \otimes h_{G_{v}}\right) \| h_{u v}\right]\right)$, where $h_{u v}$ is the hand-crafted features for the user pair $(u, v)$. The results show that our model still outperforms the baseline methods under most metrics (except Recall). Our future work is to improve our TGCE on the Recall metric when combined with the hand-crafted features.

\subsection{Discussions of Model Variants}

Since the hand-crafted features combined with all models are the same, from now on, we mainly explore the modeling ability of behavior sequence representations. This section further inspects different variants to show the effectiveness of each module in TGCE. The results are shown in Table 3.

4.6.1 Influence of Each Tier in TGCE. We first conduct ablation studies on two GNN layers, where w/o PGAT and w/o ONAN denote the variant models of TGCE by removing one tier of GNN model, respectively. The results reveal that:

Both ONAN and PGAT can improve our model performance. Removing the layer of ONAN/PGAT, TGCE decreases the performance with 6.74/0.87\% in F1 and 1.14\%/0.89\% in MRR@10. This shows that capturing high-order connections or long-range dependencies are essential for promoting the user matching accuracy. Removing PGAT drops sharply by $7.41 \%$ in Recall and improves by $4.80 \%$ in Precision of user pair classification. The potential reason is that adding shortcut edges makes denser connections which may improve the possibility of finding more potentially matched users, but at the cost of introducing noise interactions.

4.6.2 Variants of Modeling Long-range Dependency. We next perform variant experiments by applying different GNN models to capture long-range dependencies. We replace the PGAT model by GCN/GAT, achieving the variants as repl.GCN and repl.GAT.

(1) Both PGAT and GAT work better than the model GCN. GCN aggregates neighbors based on graph Laplacian, where each neighbor is treated differently according to their node degree. That means, GCN focuses more on graph-structure information. As opposed to GCNs, our model PGAT and the standard model GAT borrow the idea of attention mechanisms, and can learn to assign different weights to different neighbors in the aggregation process. Actually, in the scenario of SSGs, both original neighbors and pseudo 

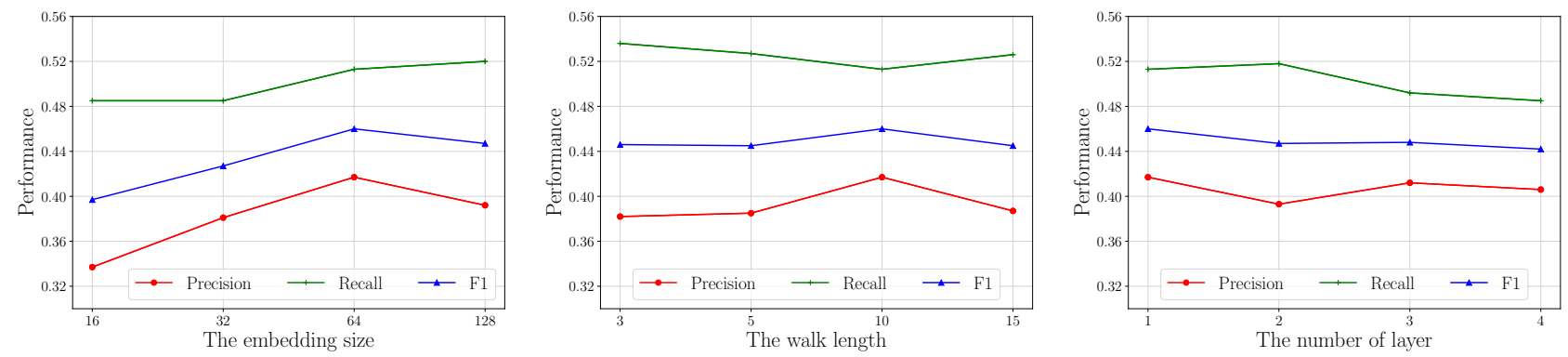

Figure 3: The hyper-parameter study of embedding size, random-walk length and the number of ONAN layers.

neighbors linked by shortcut edges will be included within 1-hop relationships. Thus, compared to the structure information, learning different weights of neighbors would be more beneficial to distinguish different neighbors, so as to capture more interactions.

(2) PGAT can further outperform GAT (except Recall in the classification setting). This demonstrates the importance of encoding behavior position information when capturing long-range dependencies. Preserving the order information makes pseudo neighbors with different walking steps more distinguishable, and will help alleviate noise information in shortcut connections. Hence, PGAT can greatly improve the model's performance in the Precision metric.

4.6.3 Variants of Similarity Score Metrics. We further compare several simple similarity score metrics of cosine and dot similarity with our attention based metrics, to investigate their effects on the performance. The results show that different score metrics indeed have different effects on the performance. The gaps between the simple metrics and ours are big. That is to say, selecting an appropriate relation score function is very important for the final results. In our model, our attention based similarity score metric is more capable of fusing the features vectors to interact more adequately, so that denser interactions can be captured.

\subsection{Hyper-parameter Study}

In this section, we investigate the effects of hyper-parameters on our model. Figure 3 shows the results of TGCE with varied hyperparameters under the classification setting.

(1) Increasing the embedding size does not always lead to better performance. A larger embedding size usually increases the performance by improving the model's learning capacity, but may lead to overfitting when the size is too large. The optimal embedding size is 64 in our setting.

(2) It's easy to see that the length of 10 makes a good tradeoff and performs the best. A length lower than that will reduce the number of shortcut connections, while a one higher than that may introduce too many less credible connections. Both hurt the performance.

(3) Setting the number of ONAN layer to 1 is enough to achieve good performance, which is better than all compared baselines.

\subsection{Analysis on Behavior Sequence Length}

We further analyze the ability of dealing with different lengths of behaviors for our approach TGCE and four best baselines. We divide all users in the testing dataset into 5 groups according to

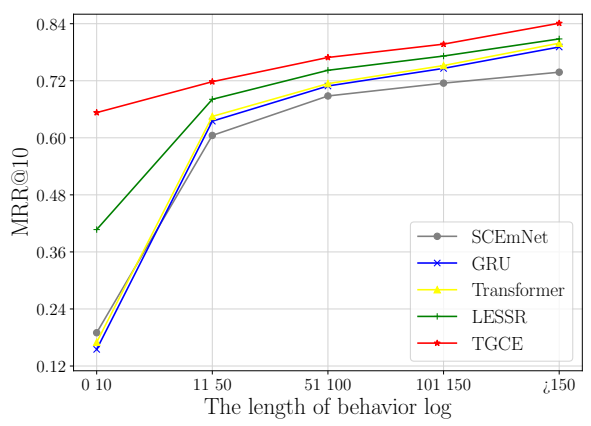

Figure 4: The performance of methods with different lengths of behavior-logs in MRR@10.

their device-logs' length, and find matched users for each one under the ranking setting. Figure 4 shows MRR@10 achieved by different methods. It can be seen that our TGCE consistently outperforms the existing baselines at different lengths. The performance gap between TGCE and other methods decreases as the length increases. This is because the length of random walks sampled in SSGs is fixed to 10 in our model, the effect of capturing long-range dependencies by PGAT will not be so obvious when the sequence length increases a lot. This also leaves a further study to future work.

\section{CONCLUSION}

This paper proposes a two-tier graph contextual embedding method called TGCE to simultaneously resolve two important issues in the cross-device user matching task, namely capturing high-order connections and long-range dependencies. The first tier is to construct behavior evolutionary graphs, and uses ONAN to preserve both sequence- and graph-structure interactions among neighbouring behaviors. The second tier is to build scaled shortcut graphs, and employs PGAT to draw global dependencies between distant behaviors. By combining the two tiers, TGCE can obtain graph contextual embeddings for behaviors, which is more meaningful to improve user matching. The experimental results show that our TGCE is powerful and outperforms various sequence modeling methods.

\section{ACKNOWLEDGMENTS}

We thank all the anonymous reviewers. Hongren Huang and Shu Guo contribute equally. This work is supported by the National Natural Science Foundation of China (No.61772151 and No.U20B2053). 


\section{REFERENCES}

[1] Abien Fred Agarap. 2018. Deep Learning using Rectified Linear Units (ReLU) CoRR abs/1803.08375 (2018). arXiv:1803.08375 http://arxiv.org/abs/1803.08375

[2] Francois Belletti, Minmin Chen, and Ed H. Chi. 2019. Quantifying Long Range Dependence in Language and User Behavior to improve RNNs. In KDD, Ankur Teredesai, Vipin Kumar, Ying Li, Rómer Rosales, Evimaria Terzi, and George Karypis (Eds.). ACM, 1317-1327. https://doi.org/10.1145/3292500.3330944

[3] Tianqi Chen and Carlos Guestrin. 2016. XGBoost: A Scalable Tree Boosting System. In KDD. 785-794. https://doi.org/10.1145/2939672.2939785

[4] Tianwen Chen and Raymond Chi-Wing Wong. 2020. Handling Information Loss of Graph Neural Networks for Session-based Recommendation. In KDD. ACM, 1172-1180. https://dl.acm.org/doi/10.1145/3394486.3403170

[5] Junyoung Chung, Çaglar Gülçehre, KyungHyun Cho, and Yoshua Bengio. 2014 Empirical Evaluation of Gated Recurrent Neural Networks on Sequence Modeling. CoRR abs/1412.3555 (2014). arXiv:1412.3555 http://arxiv.org/abs/1412.3555

[6] Aditya Grover and Jure Leskovec. 2016. node2vec: Scalable Feature Learning for Networks. In KDD. ACM, 855-864. https://doi.org/10.1145/2939672.2939754

[7] Xiangnan He, Hanwang Zhang, Min-Yen Kan, and Tat-Seng Chua. 2016. Fast Matrix Factorization for Online Recommendation with Implicit Feedback. In SIGIR. 549-558. https://doi.org/10.1145/2911451.2911489

[8] Balázs Hidasi, Alexandros Karatzoglou, Linas Baltrunas, and Domonkos Tikk. 2016. Session-based Recommendations with Recurrent Neural Networks. In ICLR. http://arxiv.org/abs/1511.06939

[9] Sepp Hochreiter and Jürgen Schmidhuber. 1997. Long Short-Term Memory. Neural Comput. 9, 8 (1997), 1735-1780. https://doi.org/10.1162/neco.1997.9.8.1735

[10] Bowen Jin, Chen Gao, Xiangnan He, Depeng Jin, and Yong Li. 2020. Multibehavior Recommendation with Graph Convolutional Networks. In SIGIR. ACM, 659-668. https://doi.org/10.1145/3397271.3401072

[11] Diederik P. Kingma and Jimmy Ba. 2015. Adam: A Method for Stochastic Opti mization. In ICLR, Yoshua Bengio and Yann LeCun (Eds.). http://arxiv.org/abs/ 1412.6980

[12] Thomas N. Kipf and Max Welling. 2017. Semi-Supervised Classification with Graph Convolutional Networks. In ICLR. https://openreview.net/forum?id= SJU4ayYgl

[13] Quoc V. Le and Tomás Mikolov. 2014. Distributed Representations of Sentences and Documents. In ICML, Vol. 32. JMLR.org, 1188-1196. http://proceedings.mlr press/v32/le14.html

[14] Chen Li, Xutan Peng, Hao Peng, Jianxin Li, Lihong Wang, and Philip S. Yu 2020. Forming an Electoral College for a Graph: a Heuristic Semi-supervised Learning Framework. CoRR abs/2006.06469 (2020). arXiv:2006.06469 https: //arxiv.org/abs/2006.06469

[15] Guohao Li, Matthias Müller, Ali K. Thabet, and Bernard Ghanem. 2019. DeepGCNs: Can GCNs Go As Deep As CNNs?. In ICCV. IEEE, 9266-9275. https: //doi.org/10.1109/ICCV.2019.00936

[16] Jing Li, Pengjie Ren, Zhumin Chen, Zhaochun Ren, Tao Lian, and Jun Ma. 2017. Neural Attentive Session-based Recommendation. In CIKM. ACM, 1419-1428. https://doi.org/10.1145/3132847.3132926

[17] Xiaoxue Li, Yanan Cao, Qian Li, Yanmin Shang, Yangxi Li, Yanbing Liu, and Guandong Xu. 2021. RLINK: Deep reinforcement learning for user identity linkage. World Wide Web 24, 1 (2021), 85-103. https://doi.org/10.1007/s11280020-00833-8

[18] Yujia Li, Daniel Tarlow, Marc Brockschmidt, and Richard S. Zemel. 2016. Gated Graph Sequence Neural Networks. In ICLR, Yoshua Bengio and Yann LeCun (Eds.). http://arxiv.org/abs/1511.05493

[19] Zhongyang Li, Xiao Ding, and Ting Liu. 2018. Constructing Narrative Event Evolutionary Graph for Script Event Prediction. In IfCAI. ijcai.org, 4201-4207. https://doi.org/10.24963/ijcai.2018/584

[20] Jianxun Lian and Xing Xie. 2016. Cross-Device User Matching Based on Massive Browse Logs: The Runner-Up Solution for the 2016 CIKM Cup. CoRR abs/1610.03928 (2016). arXiv:1610.03928 http://arxiv.org/abs/1610.03928

[21] Zhiwei Liu, Mengting Wan, Stephen Guo, Kannan Achan, and Philip S Yu. 2020. BasConv: Aggregating Heterogeneous Interactions for Basket Recommendation with Graph Convolutional Neural Network. In SDM. SIAM, 64-72.
[22] Xiongcai Luo, Jun Gao, Chang Zhou, and Jeffrey Xu Yu. 2017. UniWalk: Unidirectional Random Walk Based Scalable SimRank Computation over Large Graph. In ICDE. IEEE Computer Society, 325-336. https://doi.org/10.1109/ICDE.2017.92

[23] Sean MacAvaney, Franco Maria Nardini, Raffaele Perego, Nicola Tonellotto, Nazli Goharian, and Ophir Frieder. 2020. Efficient Document Re-Ranking for Transformers by Precomputing Term Representations. In SIGIR. ACM, 49-58. https://doi.org/10.1145/3397271.3401093

[24] Minh C. Phan, Aixin Sun, and Yi Tay. 2017. Cross-Device User Linking: URL, Session, Visiting Time, and Device-log Embedding. In SIGIR. ACM, 933-936. https://doi.org/10.1145/3077136.3080682

[25] Ruihong Qiu, Jingjing Li, Zi Huang, and Hongzhi Yin. 2019. Rethinking the Item Order in Session-based Recommendation with Graph Neural Networks. In CIKM. ACM, 579-588. https://doi.org/10.1145/3357384.3358010

[26] Chen Qu, Chenyan Xiong, Yizhe Zhang, Corby Rosset, W. Bruce Croft, and Paul Bennett. 2020. Contextual Re-Ranking with Behavior Aware Transformers. In SIGIR. ACM, 1589-1592. https://doi.org/10.1145/3397271.3401276

[27] Juan Ramos. 1999. Using TF-IDF to Determine Word Relevance in Document Queries.

[28] Badrul Munir Sarwar, George Karypis, Joseph A. Konstan, and John Riedl. 2001. Item-based collaborative filtering recommendation algorithms. In $W W W$. https: //doi.org/10.1145/371920.372071

[29] Vighnesh Leonardo Shiv and Chris Quirk. 2019. Novel positional encodings to enable tree-based transformers. In NIPS. 12058-12068. https://proceedings.neurips. cc/paper/2019/hash/6e0917469214d8fbd8c517dcdc6b8dcf-Abstract.html

[30] Fei Sun, Jun Liu, Jian Wu, Changhua Pei, Xiao Lin, Wenwu Ou, and Peng Jiang. 2019. BERT4Rec: Sequential Recommendation with Bidirectional Encoder Representations from Transformer. In CIKM. ACM, 1441-1450. https: //doi.org/10.1145/3357384.3357895

[31] Qingyun Sun, Hao Peng, Jianxin Li, Senzhang Wang, Xiangyu Dong, Liangxuan Zhao, S Yu Philip, and Lifang He. 2020. Pairwise learning for name disambiguation in large-scale heterogeneous academic networks. In 2020 IEEE International Conference on Data Mining (ICDM). IEEE, 511-520.

[32] Jiaxi Tang, Francois Belletti, Sagar Jain, Minmin Chen, Alex Beutel, Can Xu, and Ed H. Chi. 2019. Towards Neural Mixture Recommender for Long Range Dependent User Sequences. In WWW. ACM, 1782-1793. https://doi.org/10.1145/ 3308558.3313650

[33] Ugo Tanielian, Anne-Marie Tousch, and Flavian Vasile. 2018. Siamese Cookie Embedding Networks for Cross-Device User Matching. In WWW, Pierre-Antoine Champin, Fabien L. Gandon, Mounia Lalmas, and Panagiotis G. Ipeirotis (Eds.). ACM, 85-86. https://doi.org/10.1145/3184558.3186941

[34] Yi Tay, Cong-Minh Phan, and Tuan-Anh Nguyen Pham. 2016. Cross Device Matching for Online Advertising with Neural Feature Ensembles : First Place Solution at CIKM Cup 2016. CoRR abs/1610.07119 (2016). arXiv:1610.07119 http://arxiv.org/abs/1610.07119

[35] Ashish Vaswani, Noam Shazeer, Niki Parmar, Jakob Uszkoreit, Llion Jones, Aidan N. Gomez, Lukasz Kaiser, and Illia Polosukhin. 2017. Attention is All you Need. In NIPS. 5998-6008. http://papers.nips.cc/paper/7181-attention-is-allyou-need

[36] Petar Velickovic, Guillem Cucurull, Arantxa Casanova, Adriana Romero, Pietro Liò, and Yoshua Bengio. 2018. Graph Attention Networks. In ICLR. https: //openreview.net/forum?id=rJXMpikCZ

[37] Shoujin Wang, Liang Hu, Yan Wang, Longbing Cao, Quan Z. Sheng, and Mehmet A. Orgun. 2019. Sequential Recommender Systems: Challenges, Progress and Prospects. In IFCAI. ijcai.org, 6332-6338. https://doi.org/10.24963/ijcai.2019/ 883

[38] Shu Wu, Yuyuan Tang, Yanqiao Zhu, Liang Wang, Xing Xie, and Tieniu Tan. 2019. Session-Based Recommendation with Graph Neural Networks. In AAAI. AAAI Press, 346-353. https://doi.org/10.1609/aaai.v33i01.3301346

[39] Chengfeng Xu, Pengpeng Zhao, Yanchi Liu, Victor S. Sheng, Jiajie Xu, Fuzhen Zhuang, Junhua Fang, and Xiaofang Zhou. 2019. Graph Contextualized SelfAttention Network for Session-based Recommendation. In IFCAI. ijcai.org, 39403946. https://doi.org/10.24963/ijcai.2019/547

[40] Hang Yan, Bocao Deng, Xiaonan Li, and Xipeng Qiu. 2019. TENER: Adapting Transformer Encoder for Named Entity Recognition. CoRR abs/1911.04474 (2019). arXiv:1911.04474 http://arxiv.org/abs/1911.04474 